\title{
Avaliação do Potencial Antioxidante dos Extratos da Madeira do Cafeeiro Produzidos em Diferentes Sistemas de Cultivos
}

ISSN: 1984-3151

\author{
Evaluation of Antioxidant Potential of Extracts of Coffee \\ Wood Produced in Different Farming Systems
}

\author{
Jorge Luiz Peixoto Bispoㄹ; Cassiana Alves Ferreira²; Patrícia Kauanna Fonseca \\ Damasceno $^{3}$; Calila Teixeira Santos ${ }^{4}$
}

\footnotetext{
1 Doutorando em Biotecnologia. UEFS, 2017. Técnico em Alimentos do Instituto Federal de Educação, Ciência e Tecnologia Baiano. Itapetinga, BA. jorge.bispo@itapetinga.ifbaiano.edu.br.

2 Doutoranda. UFLA, 2017. Universidade Federal de Lavras UFLA. Lavras, MG. cassianaaf@gmail.com.

3 Doutoranda. UFBA-Fiocruz, 2017. Universidade Federal da Bahia- UFBA. Salvador, BA. patriciakauanna@hotmail.com.

4 Doutoranda. UEFS, 2017. Professora do Instituto Federal de Educação, Ciência e Tecnologia Baiano. Senhor do Bonfim, BA. calila.santos@bonfim.bonfim.edu.br.
}

Recebido em: 15/11/2016 - Aprovado em: 16/05/2017 - Disponibilizado em: 31/05/2017

\begin{abstract}
RESUMO: A produção nacional de café gera uma grande quantidade de resíduos como cascas e madeiras devido à substituição de plantios antigos. O estudo químico da madeira auxilia a compreender a composição dos seus constituintes e pode agregar valor ao resíduo. Assim, os objetivos deste trabalho foram avaliar o potencial antioxidante e os teores de compostos fenólicos e flavonoides dos extratos da madeira do cafeeiro de duas cultivares diferentes e produzidas em diferentes sistemas de cultivo. De acordo com os resultados, a amostra que apresentou melhores resultados foi a variedade Catuaí cultivada no sistema Natural, apresentando 28,02 mg EAG/100mg do extrato para compostos fenólicos, $18,49 \mathrm{mg} E Q / 100 \mathrm{mg}$ do extrato para flavonoides e $34,5 \%$ de sequestro do DPPH• o que sugere que está condição favorece o potencial antioxidante da madeira do cafeeiro. PALAVRAS-CHAVE: Madeira. Antioxidante. Coffea arabica.
\end{abstract}

ABSTRACT: National coffee production generates a large amount of waste such as bark and wood due to the replacement of old plantations. The chemical study of wood helps to understand the composition of its constituents and it can add value to the residue. Thus, this study aims to evaluate the antioxidant potential and the levels of phenolic compounds and flavonoids extracts of coffee wood from two different cultivars and produced in different cropping systems. According to the results, the sample which showed best results was the Catuai variety which is grown in the natural system, showing $28.02 \mathrm{mg} E A G / 100 \mathrm{mg}$ of the extract for phenolic compounds, $18.49 \mathrm{mg}$ Eq/100 $\mathrm{mg}$ of the extract to flavonoids and $34.5 \%$ of the DPPH•, which suggests that this condition favors the antioxidant potential of the wood of the coffee plant.

KEYWORDS: Wood. Antioxidant. Coffea arabica. 


\section{INTRODUÇÃo}

O Brasil é o maior produtor de café do mundo, a estimativa da área plantada em 2016 é de 2.209.097 milhões de hactares, e é 1,8\% menor do que em 2015. Sendo que $79 \%$, o que corresponde a 1.753 .100 hectares corresponde Coffea arabica L. (Rubiaceae) (COMPANHIA NACIONAL DE ABASTECIMENTO CONAB, 2016). Tamanha produção gera uma enorme quantidade de resíduos lignocelulósicos, como cascas, madeira provenientes de plantios antigos, etc. que podem, e devem ser reaproveitados de alguma forma.

Estudos recentes foram realizados com o intuito de aproveitar ao máximo os resíduos provenientes da cafeicultura, como a utilização da madeira para confecção de móveis ecológicos (PEREIRA et al., 2014), a utilização de resíduos de madeira e casca para energéticos na fabricação de briquetes (PROTÁsIO et al., 2012).

Paz Castro et al., (2015) sugere madeiras amazônicas como alternativas viáveis para o armazenamento e envelhecimento de bebidas destiladas, ressaltando as análises físico-químicas das espécies estudadas. A madeira do cafeeiro, surge, no entanto, como alternativa para $o$ armazenamento e envelhecimento de bebidas destiladas, no entanto faz-se necessário estudos acerca de suas propriedades físico-químicas.

Coffea arabica L. e Coffea canephora Pierre são duas espécies importantes na economia do café nacional e pertencem ao gênero Coffea L. família Rubiaceae. Possuem características de plantas perenes, ciclo bianual, adaptadas a climas úmidos, porte arbustivo ou arbóreo, de caule lenhoso de forma cônico-irregular com casca cinzenta e rugosa, folhas persistentes, onduladas nos bordos e de coloração verdeacinzentada, quando jovens brilhantes, quando adulta e flores brancas hermafroditas (PEREIRA, 2008). As plantações são conduzidas em sistemas de cultivos específicos para cada localidade ou tipo de solo que podem influenciar a qualidade $\mathrm{e} o$ rendimento energético da madeira do cafeeiro.

Os três sistemas de cultivos utilizados no Brasil são convencionais, no qual utilizam os preceitos clássicos da cafeicultura, o Sistema Natural, em que há pouca interferência humana, com podas regulares e não são utilizados agrotóxicos, e o Sistema Orgânico, que visam a diminuição dos resíduos químicos e uma diversificação das culturas consorciadas, preservando os recursos naturais (THEODORO et al., 2003).

Um dos maiores problemas da cafeicultura é o elevado volume de resíduo, advindo das podas e recepa baixa das plantas (OLIVEIRA, 2005). A madeira é utilizada para uso próprio como combustível ou pode ser vendida a um baixo valor. Porém, na maioria dos casos, são simplesmente abandonadas e queimadas na própria lavoura (SILVA et al., 2003). Dessa forma, o volume dos resíduos não é quantificado pelos produtores, por não serem considerados subprodutos com valor agregado (PEREIRA, 2008).

Estudos das propriedades tecnológicas da madeira são recomendados para agregar valor à determinada espécies, bem como identificar possíveis usos. O estudo da estrutura xilemática, ou seja, seus elementos celulares e seus arranjos, são indicados para o conhecimento e identificação de espécies, assim como os estudos físico-químicos são essenciais para a compreensão do comportamento da madeira a estímulos físicos (RODRIGUES et al., 2009), bem como sua composição química elementar (proporções dos elementos) e fundamentais, incluindo seus metabólitos secundários, podendo assim identifica-los, purificando-os e separá-los.

Sabe-se que a madeira, de forma geral, possui em sua composição química elementar o carbono, hidrogênio, oxigênio e nitrogênio resultando nas 
principais macromoléculas fundamentais, celulose, hemiceluloses e lignina. Há ainda os componentes minoritários, de baixo peso molecular, que são os extrativos e as substâncias minerais. Os principais grupos químicos que compreendem as substâncias de baixo peso molecular são: compostos fenólicos, terpenos, ácidos alifáticos, álcoois, e substâncias inorgânicas (KLOCK et al., 2005) os quais podem ser extraídos em água ou solventes orgânicos (SILVÉRIO et al., 2006).

A presença de compostos fenólicos em diferentes fontes vegetais e microbianas tem sido amplamente explorada na última década, pois em sua grande maioria esses têm ação antioxidante, que tem despertado o interesse da comunidade cientifica de atuarem como conservadores naturais. Isso vem de encontro à necessidade que a indústria alimentícia está tendo em substituir o uso de substâncias sintéticas por agentes naturais com capacidade antimicrobianos e/ou antioxidantes (DEVI et al., 2009).

Sendo assim, este trabalho teve como objetivo determinar 0 teor de compostos fenólicos e flavonoides totais e avaliar o potencial antioxidante dos extratos da madeira do cafeeiro de duas cultivares produzidas em diferentes sistemas de cultivo.

\section{Desenvolvimento}

\subsection{Material e Métodos}

\subsubsection{MATERIAIS}

Etanol PA, 2-difenil-1-picrilhidrazil radical $(\mathrm{DPPH} \bullet)$, padrão rutina, ácido gálico, quercetina, reagente de Folin-Ciocalteu, carbonato de sódio $\left(\mathrm{Na}_{2} \mathrm{CO}_{3}\right)$ e cloreto de alumínio $\left(\mathrm{AlCl}_{3}\right)$ foram adquiridos da Sigma-Aldrich (Steinheim, Alemanha).

\subsubsection{Material Vegetal}

As madeiras foram coletadas no campus do Instituto Federal de Educação, Ciência e Tecnologia do Sul de Minas Gerais no município de Machado (MG), latitude $21^{\circ} 40^{\prime} \mathrm{S}$, longitude $45^{\circ} 59^{\prime} \mathrm{W}$ e altitude de $873 \mathrm{~m}$.

As cultivares foram Mundo Novo e Catuaí, em diferentes sistemas de cultivo, conforme Tabela 1 a seguir.

\section{Tabela 1}

Amostras das madeiras dos cafeeiros

\begin{tabular}{c|c}
\hline Variedade & Tipo de cultivo \\
\hline Mundo Novo & Natural (MNN) \\
Mundo Novo & Orgânico (MNO) \\
Catuaí & Natural (CAN) \\
Catuaí & Convencional (CAC) \\
Catuaí & Orgânico (CAO) \\
\hline \multicolumn{2}{c}{ Fonte - Próprio autor. }
\end{tabular}

\subsubsection{OBtenção do extrato Bruto}

Cavacos da madeira do cafeeiro foram moídos em moinhos de facas e os componentes químicos foram extraídos por maceração em etanol durante $72 \mathrm{~h}$ a temperatura ambiente $\left(25^{\circ} \mathrm{C}\right)$. A mistura foi filtrada sob vácuo e o solvente foi evaporado sob pressão reduzida e temperatura controlada a $60{ }^{\circ} \mathrm{C}$ com um rotaevaporador. O rendimento dos extratos foi de $2,44 \% ; 2,28 \% ; 2,17 \% ; 0,78 \%$ e $0,51 \%$ para $M N N$, MNO, CAN, CAC e CAO, respectivamente.

\subsection{ANÁlise do Extrato Bruto}

\subsubsection{Compostos Fenólicos Totais (CFT)}

Amostras a serem testadas foram preparadas a 100 $\mu \mathrm{g} \cdot \mathrm{mL}^{-1}$ em etanol. Em uma microplaca, $20 \mu \mathrm{L}$ do extrato foram misturados com $100 \mu \mathrm{L}$ do reagente de Folin-Ciocalteu (1:10) e, após $8 \mathrm{~min}$, foram 
adicionados $80 \mu \mathrm{L}$ de carbonato de sódio a 7,5\% (m/v) à mistura. Depois de $60 \mathrm{~min}$ no escuro e a temperatura ambiente $\left(25^{\circ} \mathrm{C}\right)$, a sua absorbância foi lida a $765 \mathrm{~nm}$ utilizando o leitor de microplacas Thermo Scientific ${ }^{\mathrm{TM}}$ Multiskan $^{\mathrm{TM}}$ FC Microplate Photometer contra um branco. A concentração dos polifenóis em amostras foi derivada a partir de uma curva de calibração padrão do ácido gálico no intervalo de $10-100 \mu \mathrm{g} \cdot \mathrm{mL}^{-1}\left(\mathrm{R}^{2}=0,9951\right)$. Os dados foram expressos em $\mathrm{mg}$ de equivalente de ácido gálico (EAG) por $100 \mathrm{mg}$ do extrato seco (JARZYCKA et al., 2013).

\subsubsection{TEOR DE FLAVONOIDES TOTAIS (TFT)}

De acordo com Jarzycka et al. (2013), com algumas modificações, $50 \mu \mathrm{L}$ de cloreto de alumínio a $2 \%$ em etanol foram adicionados a $50 \mu \mathrm{L}$ do extrato diluído em etanol (100 $\left.\mu \mathrm{g} \cdot \mathrm{mL}^{-1}\right)$. O valor de absorbância foi medido ao comprimento de onda de $420 \mathrm{~nm}$, utilizando o leitor de microplacas Thermo Scientific ${ }^{\mathrm{TM}}$ Multiskan $^{\text {TM }}$ FC Microplate Photometer depois de 30 min ao abrigo da luz e a temperatura ambiente. A absorbância final de cada amostra foi comparada com uma curva padrão de quercetina no intervalo de 10$100 \mu \mathrm{g} \cdot \mathrm{mL}^{-1}(\mathrm{R} 2=0,9986)$. Os dados foram expressos em $\mathrm{mg}$ de equivalente em quercetina (EQ) por $100 \mathrm{mg}$ de extrato seco.

\subsubsection{Determinação DA Atividade}

\section{SEquestradora do Radical DPPH•}

Para avaliar a atividade antioxidante dos extratos das madeiras do cafeeiro foi utilizado o método de capacidade de captura in vitro do radical livre DPPH • (2,2, difenil-2-picrilhidrazil), de acordo com BrandWilliams, Cuvelier e Berset (1995) e Kim et al., 2012, com modificações. O comprimento de onda utilizado foi de $492 \mathrm{~nm}$. Foram utilizados rutina e ácido ascórbico (AA) como padrões.
A cada poço da microplaca de 96 poços adicionaramse $20 \mu \mathrm{L}$ das amostras ou padrões $\left(100 \mu \mathrm{g} \cdot \mathrm{mL}^{-1}\right)$ para reagir com $80 \mu \mathrm{L}$ da solução de $\mathrm{DPPH} \bullet$. O branco foi realizado usando $20 \mu \mathrm{L}$ das amostras acrescidos de $80 \mu \mathrm{L}$ de metanol. A leitura foi efetuada depois de 10 min, mantendo-se a placa em um ambiente escuro a temperatura de $22^{\circ} \mathrm{C}$. Todos os testes foram realizados em triplicada. $\mathrm{O}$ controle negativo foi feito em três repetições, colocando-se $20 \mu \mathrm{L}$ de metanol e $80 \mu \mathrm{L}$ de DPPH, a leitura foi feita imediatamente (recomendado). A percentagem de sequestro de radicais livres (\%SRL) foi calculada de acordo com a Equação 1.

$$
\% S R L=\frac{\text { Abscontrole }- \text { Absamostra }}{\text { Abscontrole }} \times 100
$$

em que: $\mathrm{SRL}=$ sequestro de radicais livres, Abscontrole $=$ absorbância do controle negativo, Absamostra = absorbância da amostra.

\subsection{ANÁLISE Estatística}

Os dados foram analisados e a comparação das médias foi feita através da ANOVA seguida do teste de Tukey a $5 \%$ de probabilidade, utilizando o software GraphPad Prism 5.0.

\section{Resultados e Discussão}

Resultados de CFT, TFT e \%SRL são apresentados na Tabela 2. Conforme os testes realizados, o teor de compostos fenólicos dos extratos da madeira do cafeeiro foi maior em CAN em comparação com os demais e os menores valores foram encontrados em MNO e CAO, extratos que não diferem estatisticamente entre si $(p<0,05)$. Para o teste de teor de flavonoides totais, os valores ficaram muito próximos entre todas as análises, sendo encontradas poucas diferenças válidas estatisticamente, segundo o teste de Tukey, o que sugere que as diferentes 
variedades e sistemas de cultivos não refletem

avaliadas.

distinção da síntese de flavonoides nas condições

Tabela 2

Teores de compostos fenólicos, flavonoides totais e atividade antioxidante dos extratos da madeira do cafeeiro nas diferentes variedades

\begin{tabular}{|c|c|c|c|}
\hline $\begin{array}{c}\text { Amostra } \\
(100 \mu \mathrm{g} / \mathrm{mL})\end{array}$ & $\begin{array}{c}\text { Compostos Fenólicos } \\
\text { (mgEAG/100mg do extrato)* }\end{array}$ & $\begin{array}{c}\text { Flavonoides (mgEQ/100mg } \\
\text { do extrato)* }\end{array}$ & $\begin{array}{c}\text { Sequestro do } \\
\text { DPPH・ }(\% S R L)^{\star}\end{array}$ \\
\hline MNN & $25,26 \pm 0,80^{a}$ & $16,79 \pm 0,05^{a}, \mathrm{~b}, \mathrm{c}, \mathrm{d}$ & 29,6 \\
\hline MNO & $22,74 \pm 0,72^{b}$ & $17,46 \pm 0,07^{a}, \mathrm{e}, \mathrm{f}, \mathrm{g}$ & 25,8 \\
\hline CAN & $28,02 \pm 0,20^{c}$ & $18,49 \pm 0,25^{\mathrm{b}, \mathrm{e}, \mathrm{h}}$ & 34,5 \\
\hline CAC & $24,92 \pm 0,40^{a}$ & $18,06 \pm 0,18^{c, f, h . i}$ & 29,5 \\
\hline $\mathrm{CAO}$ & $22,28 \pm 0,91^{b}$ & $18,49 \pm 0,14^{\mathrm{d}, \mathrm{g}, \mathrm{i}}$ & 29,6 \\
\hline Rutina** $^{*}$ & - & - & 90,1 \\
\hline$A A^{* *}$ & - & - & 91,0 \\
\hline
\end{tabular}

De acordo com a Tabela 2, os valores encontrados para o teor de compostos fenólicos variaram de 22,28 a 28,02 mg EAG/100 mg de extrato. O conteúdo fenólico pode ser relacionado diretamente com a capacidade antioxidante. $\mathrm{Na}$ literatura são citados valores médios entre 11,9 e $22,3 \mathrm{mg}$ EAG/100 mg para a madeira de Eucalyptus globulus (VÁZQUEZ et al., 2008), 3,84 e 4,53 mg de EAG/100 mg para Maclura tinctoria (LAMOUNIER, 2010), 1,09 e 30,0 mg de EAG/100 mg para Acacia auriculiformis (SINGH et al., 2007a; SINGH et al., 2007b), 4,59 mg de EAG/100 $\mathrm{mg}$ para Terminalia brasiliensis (SOUSA et al., 2007) e 3,77 e 4,38mg de EAG/100 mg para Astronium urundeuva (QUEIROZ, 2001). Portanto, os valores determinados para as madeiras do cafeeiro são superiores ou semelhantes aos encontrados por esses autores.
Os teores de flavonoides variaram de 16,79 a 18,49 mg EQ/100mg do extrato, sendo que os maiores teores de flavonoides e compostos fenólicos foram para a amostra CAN.

O teste de sequestro do radical $\mathrm{DPPH} \cdot$ para as amostras da madeira do café corrobora o teste de fenólicos totais, sendo que o maior valor encontrado foi de $34,5 \%$ de sequestro de radical livre no extrato que tem maior concentração de compostos fenólicos, CAN. Em trabalhos de Singh et al. (2007a), o extrato da casca Acacia auriculiformis apresentou 31,0\%, já Sousa et al. (2007), encontrou um sequestro de radical livre de $91,36 \%$ para o extrato da casca de Terminalia brasiliensis.

Os padrões rutina e ácido ascórbico, utilizados como controles positivos no teste, apresentaram uma inibição do radical DPPH• de 90,1 e 91,0\%, 
respectivamente, valores superiores às amostras, porém tratam-se de padrões com alto grau de pureza e antioxidantes já conhecidos. Portanto, um resíduo descartável conseguindo obter um considerável percentual de sequestro do radical pode sugerir um potencial sendo desprezado e despertar para formas de reaproveitamento.

Segundo Gobbo-Neto e Lopes (2007), a presença, bem como a intensidade dos metabólitos secundários produzidos por plantas podem ser diretamente afetadas por diversos fatores, principalmente, índice pluviométrico, altitude, temperatura, radiação UV, nutrientes do solo, composição atmosférica e herbivoria. Dessa forma, uma mesma espécie pode apresentar diferentes perfis de compostos quando cultivadas em condições distintas.

De acordo com Gershenzon (1984) condições de escassez nutricional, principalmente deficiência de nitrogênio e fósforo, podem resultar em aumento nas concentrações de metabólitos secundários. Em ambientes com menor taxa de síntese de proteínas gera desvio do carboidrato não utilizado para síntese fenólica. O cafeeiro cultivado no sistema natural não realiza adubações químicas e o fornecimento de nutrientes é mais deficiente, sendo assim, existe a possibilidade do aumento do potencial antioxidante e do teor de fenólicos ser decorrente da escassez nutricional quando comparado aos outros tratos culturais convencionais da produção do cafeeiro.

\section{Conclusão}

A amostra que apresentou resultados mais significativos foi o extrato da variedade Catuaí cultivada no sistema Natural, que é isento de adubações químicas, o que sugere aumento na síntese de metabólitos secundários em situações de escassez nutricional. No entanto, as outras amostras também apresentaram potencial antioxidante e conteúdo fenólico, o que desperta para estudos mais aprofundados na intenção de investigar melhor seus potenciais e reaproveitar estes resíduos que atualmente são desprezados.

\section{AGRADECIMENTOS}

Ao Instituto Federal de Educação, Ciência e Tecnologia Baiano (IF BAIANO), Fundação de Amparo à Pesquisa do Estado da Bahia (FAPESB), Coordenação de Aperfeiçoamento de Pessoal de Nível Superior (CAPES) pelo apoio financeiro e bolsas concedidas.

\section{REFERÊNCIAS}

BRAND-WILLIAMS, W.; CUVELIER, M.E.; BERSET, C. Use of a free radical method to evaluate antioxidant activity. LWT- Food Science and Technology, n. 28, v. 1 , p. 25-30, 1995.

CONAB - COMPANHIA NACIONAL DE

ABASTECIMENTO. Segundo levantamento safra de café em 2016. Disponível em

http://www.conab.gov.br/OlalaCMS/uploads/arquivos/1 6_06_10_15_13_24_boletim_cafe_-_maio_2016.pdf

DEVI, M. K. A. et al.. Functional attributes of soybean seeds andproducts, with reference to isoflavone content and antioxidant activity. Food Chemistry, v. 114, n 3, p. 771-776, 2009.

GERSHENZON, J. Changes in the Levels of Plant Secondary Metabolite Production Under Water and Nutrient Stress. Recent Advances in

Phytochemistry, v. 18, p. 273-320, 1984.

GOBBO-NETO, L.; LOPES, N. P. Plantas medicinais: fatores de influência no conteúdo de metabólitos secundários. Química Nova, v. 30, n. 2, 374-381, 2007. 
JARZYCKA, A. et al.. Assessment of extracts of Helichrysum arenarium, Crataegus monogyna, Sambucus nigra in photoprotective UVA and UVB; photostability in cosmetic emulsions. Journal of Photochemistry and Photobiology. B: Biology, v.128, p. 50-57, 2013.

KIM, J; MOON, S. H.; AHN, D. U.; PAIK, H. D.; PARK, E. Antioxidant effects of ovotransferrin and its hydrolysates. Poultry Science, v. 91, n. 11, p. 27472754, 2012.

KLOCK, U. et al.. Química da madeira. 3.ed. Curitiba: UFP. Setor de Ciências Agrárias. 86p., 2005.

LAMOUNIER, K. C. Estudo dos polifenóis, atividade antioxidante e antimicrobiana da madeira e casca de Maclura tinctoria (L.) D. Don ex Steud. 2010. 113 p. Dissertação (Mestrado em Química) - Instituto de Química, Universidade Federal de Uberlância, Uberlândia.

OLIVEIRA, A. S. Casca de café ou casca de soja em substituição ao milho em dietas à base de cana de açúcar para vacas leiteiras. 2005. 109 f. Tese (Mestrado em Zootecnia) - Universidade Federal de Viçosa, Viçosa.

PAZ CASTRO, J. et al.. Uso de espécies Amazônicas para envelhecimento de bebidas destiladas: Análise Física e Química da Madeira. Cerne, v. 21, n. 2, p. 319-327, 2015.

PEREIRA, M. A. Confecções de móveis com a madeira de Coffea arábica L. 2008. 59 p.

Dissertação (Mestrado em Ciências e Tecnologia da Madeira) - Universidade Federal de Lavras, Lavras.

PEREIRA, M. A. et. al.. Propriedades anatômicas, químicas e de densidade da madeira de Coffea arábica L. Cerne. v. 20 n. 3, p. 363-367, 2014.

PROTÁSIO, T. P. et al.. Torrefação e carbonização de briquetes de resíduos do processamento dos grãos de café. Revista Brasileira de Engenharia Agrícola e Ambiental. v. 16, n. 11, p. 1252-1258, 2012.
QUEIROZ, C. R. A. dos A. Análise da lignina e dos polifenóis da Aroeira-Preta (Astronium urundeuva). 2001. 175 p. Dissertação (Mestrado em Química) - Instituto de Química, Universidade Federal de Uberlância.

RODRIGUES, B. P. et al.. Caracterização anatômica do lenho do cafeeiro (Coffea arabica L.) proveniente da região do sul do Espírito Santo. XII Encontro Latino Americano de Iniciação Científica e VIII Encontro Latino Americano de Pós-Graduação Universidade do Vale do Paraíba, 2009.

SILVA, A. L. da; FARIA, M. A. de; REIS, R. P. Viabilidade técnico-econômica do uso do sistema de irrigação por gotejamento na cultura do cafeeiro.

Revista Brasileira de Engenharia Agrícola e Ambiental, Campina Grande, v. 7, n.1, p. 37-44, jan./abr. 2003.

SILVÉRIO, F. O. et al.. Metodologia de Extração e Determinação do Teor de Extrativos em Madeiras de Eucalipto. Revista Árvore, Viçosa, v.30, n.6, p. 10091016, 2006.

SINGH, R. et al.. Free radical-scavenging activity of acetone extract/fractions of Acacia auriculiformis A. Cunn. Food Chemistry, v. 103, p. 1403-1410, 2007a.

SINGH, R. et al.. Studies on antioxidant potential of methanol extract/fractions of Acacia auriculiformis A. Cunn. Food Chemistry, v. 103, p. 505-511, 2007b.

SOUSA, C. M. M. et al.. Fenóis totais e atividade antioxidante de cinco plantas medicinais. Química Nova, v. 30, no. 2, p. 351-355, 2007.

THEODORO, V. C. A. et al.. Alterações químicas em solo submetido a diferentes formas de manejo do cafeeiro. Revista Brasileira de Ciência do Solo, Viçosa, v. 27, p. 1039-1047, 2003.

VÁZQUEZ, G. et al.. Antioxidant activity and phenolic content of chestnut (Castanea sativa) shell and eucalyptus (Eucalyptus globulus) bark extracts. Industrial Crops and Products, v. 28 p. 279-285, 2008. 